\title{
Accuracy and Diversity of Wearable Device-Based Gait Speed Measurement Among Older Men: Observational Study
}

Min-gu Kang ${ }^{1}$, MSc, MD; Seong-Ji Kang ${ }^{2,3}$, MSc, MD; Hye-Kang Roh ${ }^{3}$, MSc; Hwa-Young Jung ${ }^{3}$, MSc; Sun-wook $\mathrm{Kim}^{4}$, MSc, MD; Jung-Yeon Choi ${ }^{4}, \mathrm{MD}, \mathrm{PhD}$; Kwang-il Kim ${ }^{4,5}, \mathrm{MD}, \mathrm{PhD}$

\footnotetext{
${ }^{1}$ Department of Internal Medicine, Chonnam National University Bitgoeul Hospital, Gwangju, Republic of Korea

${ }^{2}$ Graduate School of Health Science and Management, Yonsei University, Seoul, Republic of Korea

${ }^{3}$ WELT Corp, Ltd, Seoul, Korea, Seoul, Republic of Korea

${ }^{4}$ Department of Internal Medicine, Seoul National University Bundang Hospital, Seongnam, Republic of Korea

${ }^{5}$ Department of Internal Medicine, Seoul National University College of Medicine, Seoul, Republic of Korea
}

\section{Corresponding Author:}

Kwang-il Kim, MD, PhD

Department of Internal Medicine

Seoul National University Bundang Hospital

82 Gumi-Ro 173 Beon-Gil, Seongnamsi Gyeonggi-Do

Seongnam, 13620

Republic of Korea

Phone: 82317877032

Email: kikim907@snu.ac.kr

\section{Abstract}

Background: Gait speed measurements are widely used in clinical practice, as slow gait is a major predictor of frailty and a diagnostic criterion for sarcopenia. With the development of wearable devices, it is possible to estimate the gait speed in daily life by simply wearing the device.

Objective: This study aims to accurately determine the characteristics of daily life gait speed and analyze their association with sarcopenia.

Methods: We invited community-dwelling men aged $>50$ years who had visited the outpatient clinic at a tertiary university hospital to participate in the study. Daily life gait speed was assessed using a wearable smart belt (WELT) for a period of 4 weeks. Data from participants who wore the smart belt for at least 10 days during this period were included. After 4 weeks, data from a survey about medical and social history, usual gait speed measurements, handgrip strength measurements, and dual-energy x-ray absorptiometry were analyzed.

Results: A total of 217,578 daily life gait speed measurements from 106 participants (mean age 71.1, SD 7.6 years) were analyzed. The mean daily life gait speed was 1.23 (SD 0.26) $\mathrm{m} / \mathrm{s}$. The daily life gait speed of the participants varied according to the time of the day and day of the week. Daily life gait speed significantly slowed down with age $(P<.001)$. Participants with sarcopenia had significantly lower mean daily life gait speed (mean 1.12, SD $0.11 \mathrm{~m} / \mathrm{s}$ ) than participants without sarcopenia (mean $1.23, \mathrm{SD} 0.08 \mathrm{~m} / \mathrm{s} ; P<.001$ ). Analysis of factors related to mean daily life gait speed showed that age and skeletal muscle mass of the lower limbs were significantly associated characteristics.

Conclusions: More diverse and accurate information about gait speed can be obtained by measuring daily life gait speed using a wearable device over an appropriate period, compared with one-time measurements performed in a laboratory setting. Importantly, in addition to age, daily life gait speed is significantly associated with skeletal muscle mass of the lower limbs.

(J Med Internet Res 2021;23(10):e29884) doi: 10.2196/29884

\section{KEYWORDS}

gait speed; sarcopenia; skeletal muscle mass; wearable device 


\section{Introduction}

\section{Background}

Globally, populations are aging, including in Korea, where the population is aging rapidly because of extended life expectancy [1] and a low fertility rate. In 2017 , more than $14 \%$ of the total population in Korea was aged $\geq 65$ years.

Frailty, as a reflection of decreased physiological reserve, is closely associated with increased biological age [2], concurrent medical conditions, morbidity, and decreased survival in older individuals [3]. Frailty assessments are clinically useful for determining the heterogeneous health status of older individuals [4]. Sarcopenia, defined as low muscle mass and low muscle strength, is a key characteristic of frailty [5-7]. Recently, the treatment of sarcopenia has become a key strategy for preventing and overcoming frailty [8].

Slow gait speed is a major feature of frailty [9] and a diagnostic criterion for sarcopenia [6,7]; therefore, gait speed measurement is widely used to assess frailty. However, gait speed measurement methods are not completely standardized, and the term usual gait speed refers to a measure that has been commonly used as part of this fast, safe, and inexpensive assessment $[10,11]$. Usual gait speed is calculated by instructing the individual to walk a certain distance at their usual pace in the laboratory and then measuring the time it takes to cover that distance.

Currently, technology is advancing at a rapid pace in the area of wearable devices, which can be used to measure a variety of attributes. Wearable sensors allow frequent and continuous measurements of body functions, including various physical activities, such as walking, running, and biking [12]. With the availability of these wearable devices, it is possible to measure and track daily life gait speed in the real world without significant additional effort by asking the patient to wear the device $[13,14]$. Moreover, it is questionable whether the usual gait speed measured in the laboratory can represent daily gait speed in an individual's real world [15]. Few studies have compared usual gait speed and real-world gait speed in analyses of the associations between muscle mass or muscle strength and gait speed.

\section{Objectives}

The primary aim of this study was to identify the characteristics of daily life gait speed in community-dwelling older male adults and to analyze the association of these characteristics with sarcopenia. The secondary objective was to compare the results of daily life gait speed obtained using a wearable device with usual gait speed measured in the laboratory by analyzing the associations of muscle mass and muscle strength with gait speed.

\section{Methods}

\section{Study Population}

This observational study was conducted at Seoul National University Bundang Hospital. Men aged >50 years who could walk unassisted were recruited consecutively from November 16, 2018, to April 12, 2019.

\section{Study Protocol}

The WELT (WELT Corp, Ltd) is a belt-type wearable device that uses a triaxial accelerometer to continuously measure the wearer's gait speed while walking. The smart belt measures the walking speed using the step interval time and stride length. An algorithm for detecting and analyzing peaks is used to identify the steps. Two consecutive peaks are detected as steps occur, and the step interval time between the peaks is measured in 0.1 -second increments. There are several ways to estimate stride length, and WELT adopts a method that uses a sex-based constant and height $[16,17]$. As a result, it is possible to continuously measure gait speed that changes with the step interval time.

Participants were asked to wear the WELT for 4 weeks as they went about their daily lives, and their daily life gait speed was recorded. After 4 weeks, a survey that included questions about social history and past medical history was administered, usual gait speed and handgrip strength measurements were obtained, and a dual-energy $\mathrm{x}$-ray absorptiometry (DEXA) was performed.

Usual gait speed was calculated for each participant using distance in meters and time in seconds. A marked walkway and an automated laser-gated chronometer attached to the wall were used to calculate gait speed [18]. The walkway consisted of a $1-\mathrm{m}$ acceleration phase, 4.5-m timed section, and a $1-\mathrm{m}$ deceleration phase. The chronometer started and stopped automatically as each participant crossed into and out of the timed section. The participants were instructed to walk at their usual pace. Handgrip strength was measured using a Jamar Plus digital hand dynamometer (Patterson Medical). Participants were seated in a chair with their shoulder adducted and neutrally rotated, their elbow flexed to $90^{\circ}$, and their forearm and wrist neutrally positioned. A total of three consecutive measurements of the dominant hand were taken with a brief rest between measurements, and the average scores were obtained and recorded in kilograms. DEXA was used to measure the limb skeletal muscle mass of the participants. It is very useful for body composition analysis because fat mass, nonbone lean mass, and bone mineral content can be differentiated at both the regional and whole-body levels [19]. A Horizon W machine (Hologic Inc) was used to measure appendicular skeletal muscle mass [20]. Before the DEXA scan, participants were asked to remove all metal objects and change into hospital gowns. Scanning was performed by an imaging technician in the laboratory. The scan time was approximately 10 minutes. Appendicular skeletal muscle mass was calculated as the sum of the muscle mass of both arms and legs.

Sarcopenia was defined based on the diagnostic criteria of the Asian Working Group for Sarcopenia [6]. Low muscle strength was defined as a handgrip measurement of $<28 \mathrm{~kg}$. Low physical performance was defined as a usual gait speed of $<1.0 \mathrm{~m} / \mathrm{s}$. Sarcopenia was diagnosed when the individual demonstrated a low appendicular skeletal muscle mass and low muscle strength or low physical performance.

Daily life gait speed was analyzed using data from participants who wore the wearable device for at least 10 days during the 4-week study period. The characteristics of daily life gait speed in the real world were identified, and the association between 
daily life gait speed and sarcopenia was analyzed. In addition, usual gait speed and daily life gait speed were compared by an analysis of the association between muscle mass or muscle strength and gait speed.

\section{Statistical Analysis}

All statistical analyses were performed using SPSS version 21.0 (IBM Corporation). Continuous variables were expressed as mean (SD) and were compared using either a two-tailed unpaired $t$ test or one-way analysis of variance. Discrete variables were expressed as counts and percentages, and the proportions were compared using the chi-square test or Fisher exact test. We used correlation analysis and linear regression analysis to identify factors related to daily life gait speed. All statistical analyses were two tailed, and $P<.05$ were considered statistically significant.

\section{Ethical Standards}

This study was approved by the institutional review board of Seoul National University Bundang Hospital (Institutional
Review Board No. B-1808/486-002). All participants provided written informed consent before participation. The study was performed in accordance with the principles of the Declaration of Helsinki.

\section{Results}

\section{Characteristics of Study Participants}

A total of 217,578 daily life gait speed measurements were analyzed from 106 participants who completed the study (Figure 1). The average age of the participants was 71.1 (SD 7.6; range 52-90) years, and the average number of gait speed measurements per participant was 2052.6 (SD 1022.3; range 618-4783). The mean daily life gait speed of the participants was 1.23 (SD 0.26; range 0.94-1.39) $\mathrm{m} / \mathrm{s}$, and the average walking time per day was 88.0 (SD 40.2; range 30.5-176.5) minutes.

Figure 1. Flow diagram of the study population. SNUBH: Seoul National University Bundang Hospital.

Total participants registered in SNUBH
from November 16, 2018 to April 12, 2019
$(n=113)$

\begin{tabular}{|l|l|}
\hline & Excluded $(n=7)$ \\
1) Dropout $(n=2)$ \\
2) No measured skeletal muscle mass or handgrip strength $(n=2)$ \\
3) Insufficient gait speed data $(n=3)$
\end{tabular}

\section{Participants included in the analysis$$
(n=106)
$$

\section{Characteristics of Daily Life Gait Speed Measured Using a Wearable Device}

The characteristics of daily life gait speed were presented in Figure 2. The daily life gait speed was significantly lower in older individuals $(P<.001)$. Participants walked the fastest between $5 \mathrm{AM}$ and $7 \mathrm{AM}$ and the slowest at night $(P<.001)$. There was a statistically significant difference in daily life gait speed according to the day of the week $(P<.001)$, and weekday gait speed (mean 1.23 , SD $0.26 \mathrm{~m} / \mathrm{s}$ ) was significantly faster than weekend gait speed (mean 1.22, SD $0.26 \mathrm{~m} / \mathrm{s} ; P<.001$ ). Walking time per day differed significantly on weekdays (mean 93. 4, SD 47.0 minutes) and weekends (mean 74.5, SD 41.6 minutes; $P<.001)$.

To compare daily life gait speed with usual gait speed, the daily life gait speed for each participant was calculated as a percentile compared with their own usual gait speed. The participants' daily life gait speed varied widely. The corresponding percentiles ranged from 30.3 percentile to 181.0 percentile, with a median value of 102.9 percentile. In their daily lives, a walking speed $>25 \%$ faster than their own usual gait speed was observed in $13.46 \%(29,279 / 217,578)$ of the total number of measurements, and a walking speed $>25 \%$ slower than their own usual gait speed was observed in $14.58 \%(31,728 / 217,578)$ of the total number of measurements. In the analysis of the correlation between daily life gait speed and usual gait speed, only a negligible correlation was confirmed (Pearson $r=0.155$; $P<.001$; Figure 3 ). However, in the analysis of the correlation between mean daily life gait speed and usual gait speed, a moderate positive correlation was confirmed (Pearson $r=0.504$; $P<.001$; Figure 3 ). In the analysis of the correlation between the SD of daily life gait speed and usual gait speed, only a negligible correlation was confirmed (Pearson $r=0.195 ; P=.046$; Figure 3$)$. In addition, no significant correlation was found 
between the coefficient of variation of daily life gait speed and $\quad$ usual gait speed (Pearson $r=-0.139 ; P=.16$; Figure 3).

Figure 2. The characteristics of daily life gait speed measured using a wearable device. Comparison of daily life gait speed (A) by age group; (B) by time of day; (C) by day of the week; (D) between weekdays and weekends.

A

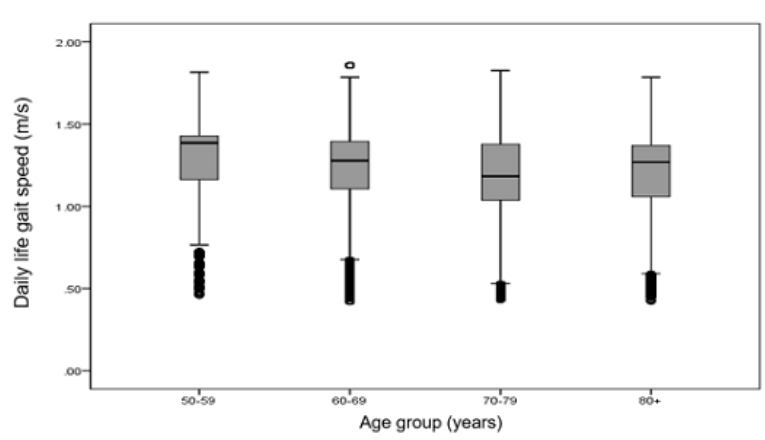

C

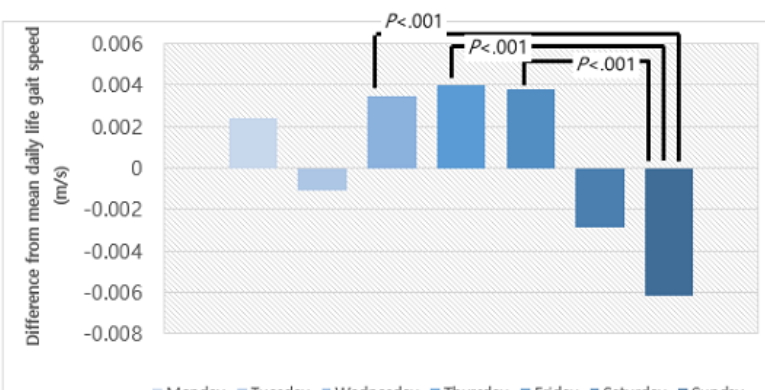

B

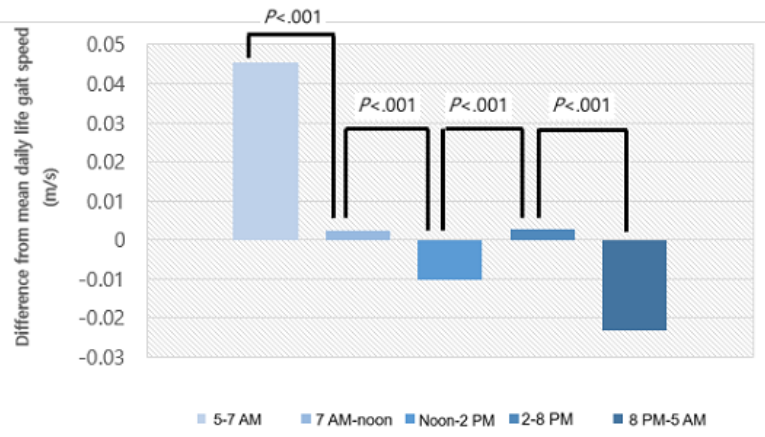

D

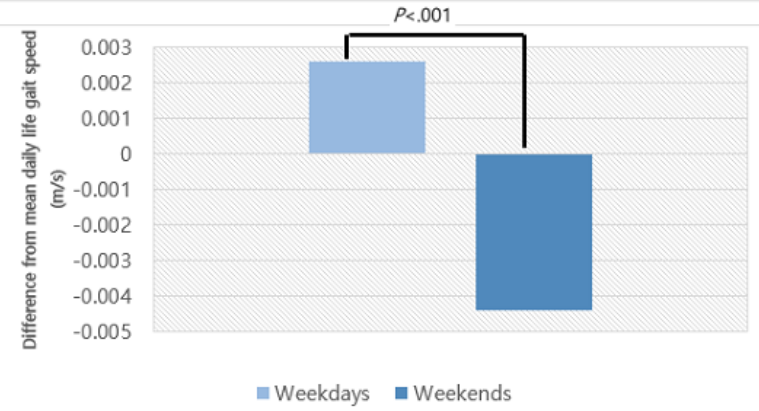

Figure 3. Scatterplot of daily life gait speed and usual gait speed. (A) Scatterplot of daily life gait speed and usual gait speed (Pearson correlation coefficient $=0.155 ; P<.001)$. (B) Scatterplot of mean daily life gait speed and usual gait speed (Pearson correlation coefficient $=0.504 ; P<.001)$. $(\mathrm{C})$ Scatterplot of SD of daily life gait speed and usual gait speed (Pearson correlation coefficient $=0.195 ; P=.046$ ). (D) Scatterplot of coefficient of variation of daily life gait speed and usual gait speed (Pearson correlation coefficient $=-0.139 ; P=.16$ ).

A

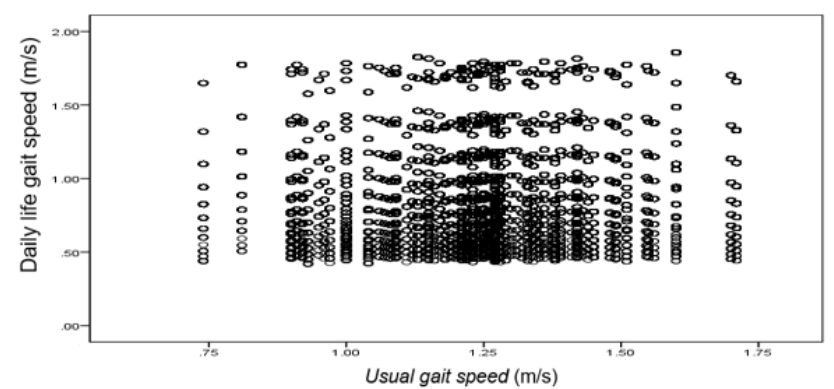

C

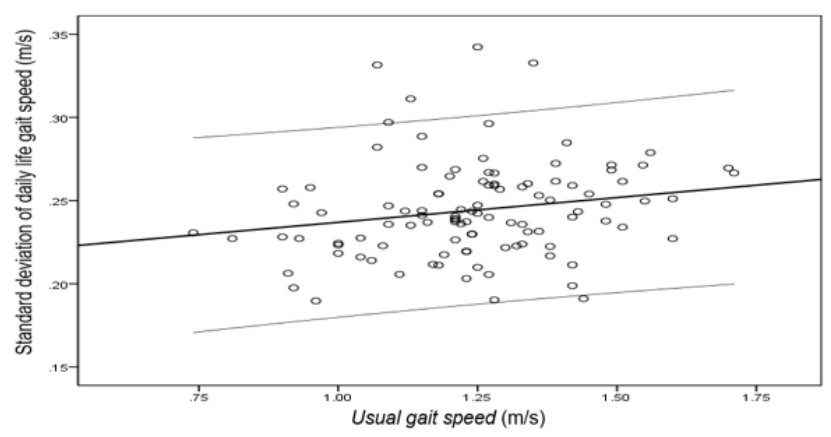

\section{Comparison Between Study Participants With and Without Sarcopenia}

Participants with low muscle strength (handgrip strength $<28$ $\mathrm{kg}$ ) had significantly lower daily life gait speed than those with

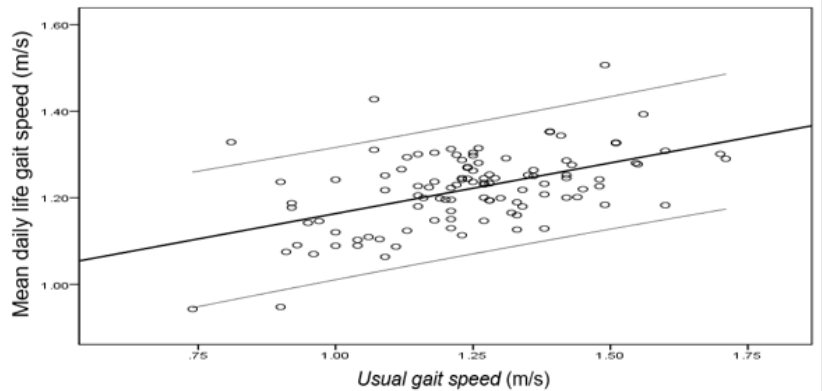

$\mathrm{D}$

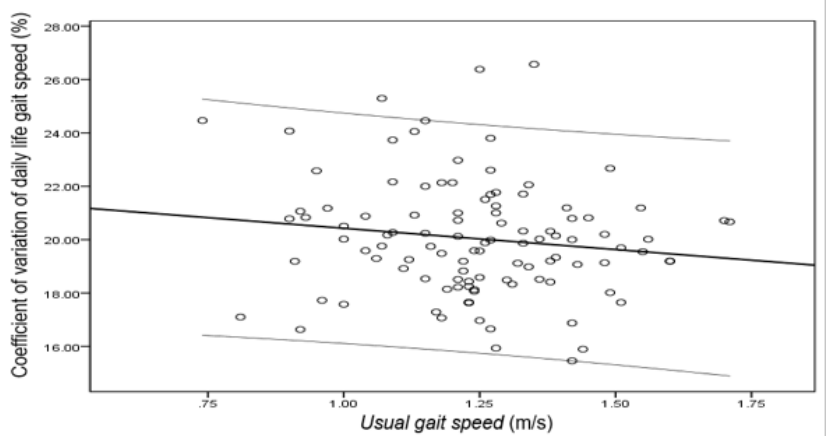

normal muscle strength (mean $1.15, \mathrm{SD} 0.25 \mathrm{~m} / \mathrm{s}$ vs mean 1.23 , SD $0.26 \mathrm{~m} / \mathrm{s} ; P<.001)$. There were also significant differences in daily life gait speed between participants with low muscle mass (appendicular skeletal muscle mass $<7.0 \mathrm{~kg} / \mathrm{m}^{2}$ ) and those 
with normal muscle mass (mean 1.22 , SD $0.26 \mathrm{~m} / \mathrm{s}$ vs mean $1.25, \mathrm{SD} 0.26 \mathrm{~m} / \mathrm{s} ; P<.001)$.

A total of 13 participants $(13 / 106,12.3 \%)$ were diagnosed with sarcopenia based on the diagnostic criteria of the Asian Working Group for Sarcopenia. Participants with sarcopenia (mean 76.0, SD 6.2 years) were older than participants without sarcopenia (mean 70.5, SD 7.6 years; $P=.01$ ) and had significantly lower mean daily life gait speed than normal participants (mean 1.12, SD $0.11 \mathrm{~m} / \mathrm{s}$ vs mean 1.23 , SD $0.08 \mathrm{~m} / \mathrm{s} ; P<.001)$. Sarcopenic participants had significantly lower handgrip strength and lower appendicular skeletal muscle mass. In addition, among participants with sarcopenia, the proportion of patients who had angina and were current smokers was significantly higher (Table 1).

Table 1. Comparison between study participants with and without sarcopenia.

\begin{tabular}{|c|c|c|c|c|}
\hline Characteristics & Total $(\mathrm{N}=106)$ & Sarcopenia $(n=13)$ & Normal $(n=93)$ & $P$ value \\
\hline Age (years) & & & & .01 \\
\hline Mean (SD) & $71.1(7.6)$ & $76.0(6.2)$ & $70.5(7.6)$ & \\
\hline Range & $52-90$ & $67-88$ & $52-90$ & \\
\hline $\operatorname{BMI}\left(\mathrm{kg} / \mathrm{m}^{2}\right)$ & & & & .54 \\
\hline Mean (SD) & $24.6(2.5)$ & $25.0(2.5)$ & $24.5(2.5)$ & \\
\hline Range & $17.0-30.8$ & $20.7-29.2$ & $17.0-30.8$ & \\
\hline Education period (years) & & & & .38 \\
\hline Mean (SD) & $14.3(3.2)$ & $13.5(3.3)$ & $14.4(3.2)$ & \\
\hline Range & $6-20$ & $8-20$ & $6-20$ & \\
\hline Job status & & & & .79 \\
\hline Incumbent & $44(41.5)$ & $5(38.5)$ & $39(41.9)$ & \\
\hline Retired & $61(57.5)$ & $8(61.5)$ & $53(57)$ & \\
\hline Hypertension, n (\%) & $67(63.2)$ & $11(84.6)$ & $56(60.2)$ & .13 \\
\hline Diabetes mellitus, n (\%) & $28(26.4)$ & $3(23.1)$ & $25(26.9)$ & .99 \\
\hline Angina, n (\%) & $22(20.8)$ & $6(46.2)$ & $16(17.2)$ & .03 \\
\hline Arthritis, n (\%) & $18(17)$ & $2(15.4)$ & $16(17.2)$ & .99 \\
\hline Smoking, n (\%) & & & & .004 \\
\hline Current smoker & $16(15.1)$ & $6(46.2)$ & $10(10.8)$ & \\
\hline Ex-smoker & $76(71.7)$ & $6(46.2)$ & $70(75.3)$ & \\
\hline Never smoker & $14(13.2)$ & $1(7.7)$ & $13(14)$ & \\
\hline Usual gait speed $(\mathrm{m} / \mathrm{s})$ & & & & $<.001$ \\
\hline Mean (SD) & $1.24(0.19)$ & $0.97(0.12)$ & $1.28(0.16)$ & \\
\hline Range & $0.74-1.71$ & $0.74-1.20$ & $0.91-1.71$ & \\
\hline Daily life gait speed $(\mathrm{m} / \mathrm{s})$ & & & & $<.001$ \\
\hline Mean (SD) & $1.22(0.09)$ & $1.12(0.11)$ & $1.23(0.08)$ & \\
\hline Range & $0.94-1.39$ & $0.94-1.33$ & $1.06-1.39$ & \\
\hline Handgrip strength $^{\mathrm{a}}(\mathrm{kg})$ & & & & $<.001$ \\
\hline Mean (SD) & $36.0(6.6)$ & $27.4(5.4)$ & $37.2(5.8)$ & \\
\hline Range & $22.1-50.3$ & $22.1-38.0$ & $24.5-50.3$ & \\
\hline Appendicular skeletal muscle mass $/ \mathrm{height}^{2}\left(\mathrm{~kg} / \mathrm{m}^{2}\right)$ & & & & .003 \\
\hline Mean (SD) & $6.74(0.74)$ & $6.39(0.34)$ & $6.79(0.77)$ & \\
\hline Range & $5.21-8.87$ & $5.68-6.97$ & $5.21-8.87$ & \\
\hline
\end{tabular}

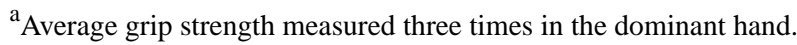

Participants with sarcopenia walked faster from 5 AM to 7 AM than at other times of the day $(P<.001)$; however, there were no significant differences between weekday and weekend gait speeds $(P=.64)$. Participants with sarcopenia (mean 78.2, SD 
45.4 minutes) had significantly less walking time per day than participants without sarcopenia (mean 89.3, SD 46.3 minutes; $P=.03$ ). In addition, there was no significant difference in walking time per day between weekdays (mean 82.4, SD 46.3 minutes) and weekends (mean 67.8, SD 42.1 minutes) in the participants with sarcopenia $(P=.17)$. In the analysis of the correlation between daily walking time and sarcopenia-related factors, it was observed that grip strength, skeletal muscle mass of the lower limbs, and usual gait speed had a negligible correlation with walking time (walking time and grip strength: Pearson $r=0.111 ; P=.003$; walking time and skeletal muscle mass of the lower limbs: Pearson $r=0.152 ; P<.001$; and walking time and usual gait speed: Pearson $r=0.112 ; P=.002)$. Analysis of the correlation between daily walking time and mean daily life gait speed revealed a low positive correlation (Pearson $r=0.315 ; P<.001)$.

There was no significant difference between the mean daily life gait speed (mean 1.12, SD $0.11 \mathrm{~m} / \mathrm{s}$ ) and the median value of daily life gait speed (mean 1.11, SD $0.12 \mathrm{~m} / \mathrm{s} ; P=.45$ ) in participants with sarcopenia, but those without sarcopenia had median values (mean 1.27 , SD $0.13 \mathrm{~m} / \mathrm{s}$ ) significantly higher than the mean values (mean $1.23, \mathrm{SD} 0.08 \mathrm{~m} / \mathrm{s} ; P<.001$ ). There was no significant difference in the SD of daily life gait speed in participants with sarcopenia (mean $0.23, \mathrm{SD} 0.02 \mathrm{~m} / \mathrm{s}$ ) and those without sarcopenia (mean 0.25 , SD $0.03 \mathrm{~m} / \mathrm{s} ; P=.06$ ). There was also no significant difference in the coefficient of variation in daily life gait speed between participants with sarcopenia (mean $20.58 \%$, SD $2.37 \%$ ) and those without sarcopenia (mean 19.96\%, SD 2.13\%; $P=.34$ ).

\section{Factors Associated With Mean Daily Life Gait Speed}

The correlation analysis of the potential factors associated with mean daily life gait speed showed that age, height, and skeletal muscle mass of the lower limbs were significantly correlated with mean daily life gait speed (Table 2). In a linear regression analysis with correlated factors, age and lower skeletal muscle mass were significantly associated with mean daily life gait speed. Participants who were younger and had more skeletal muscle mass in their lower limbs walked faster (Table 3).

Table 2. Correlation analysis (Pearson $r$ and one/two-tailed $P$ values) with mean daily life gait speed. ${ }^{\text {a }}$

\begin{tabular}{|c|c|c|c|c|c|c|}
\hline Variable & $\begin{array}{l}\text { Mean daily life } \\
\text { gait speed }\end{array}$ & Age & Height & Body weight & BMI & $\begin{array}{l}\text { Skeletal muscle mass of } \\
\text { the lower limbs }\end{array}$ \\
\hline \multicolumn{7}{|c|}{ Mean daily life gait speed } \\
\hline$r$ & 1 & -0.357 & 0.333 & 0.108 & -0.074 & 0.383 \\
\hline$P$ value & _ b & $<.001$ & $<.001$ & .27 & .45 & $<.001$ \\
\hline \multicolumn{7}{|l|}{ Age } \\
\hline$r$ & -0.357 & 1 & -0.274 & -0.237 & -0.073 & -0.338 \\
\hline$P$ value & $<.001$ & - & .004 & .01 & .46 & $<.001$ \\
\hline \multicolumn{7}{|l|}{ Height } \\
\hline$r$ & 0.333 & -0.274 & 1 & 0.466 & -0.062 & 0.523 \\
\hline$P$ value & $<.001$ & .004 & - & $<.001$ & .53 & $<.001$ \\
\hline \multicolumn{7}{|l|}{ Body weight } \\
\hline$r$ & 0.108 & -0.237 & 0.466 & 1 & 0.824 & 0.797 \\
\hline$P$ value & .27 & .01 & $<.001$ & - & $<.001$ & $<.001$ \\
\hline \multicolumn{7}{|l|}{ BMI } \\
\hline$r$ & -0.074 & -0.073 & -0.062 & 0.824 & 1 & 0.596 \\
\hline$P$ value & .45 & .46 & .53 & $<.001$ & - & $<.001$ \\
\hline \multicolumn{7}{|c|}{ Skeletal muscle mass of the lower limbs } \\
\hline$r$ & 0.383 & -0.338 & 0.523 & 0.797 & 0.596 & 1 \\
\hline$P$ value & $<.001$ & $<.001$ & $<.001$ & $<.001$ & $<.001$ & - \\
\hline
\end{tabular}

${ }^{\mathrm{a}}$ The correlation is significant at a level of .001 (one/two-tailed).

${ }^{\mathrm{b}}$ Not applicable. 
Table 3. Linear regression analysis. ${ }^{\text {a }}$

\begin{tabular}{llllll}
\hline Independent variable & $\mathrm{B}$ & $\beta$ & $t$ statistic & $P$ value & Variance inflation factor \\
\hline Constant & 0.837 & N/A & N/A & N/A & 1.146 \\
Age (years) & -0.003 & -.240 & -2.566 & .01 & .15 \\
Height (m) & 0.259 & .150 & 1.454 & .397 \\
Skeletal muscle mass of the lower limbs (kg) & 0.011 & .223 & 2.116 & .04 & 1.459 \\
\hline
\end{tabular}

${ }^{\mathrm{a}}$ Dependent variable is mean daily life gait speed.

${ }^{\mathrm{b}} \mathrm{N} / \mathrm{A}$ : not applicable.

\section{Comparison Between Daily Life Gait Speed and Usual Gait Speed in the Analysis of Associations With Muscle Mass and Muscle Strength}

A correlation analysis of gait speed, handgrip strength, and appendicular skeletal muscle mass showed that mean daily life gait speed was significantly and positively correlated with handgrip strength (Pearson $r=0.380 ; P<.001$ ) and appendicular skeletal muscle mass (Pearson $r=0.355 ; P<.001$ ). Usual gait speed had a significant positive correlation with handgrip strength (Pearson $r=0.501 ; P<.001$ ) but was less correlated with muscle mass (Pearson $r=0.227 ; P=.02$ ).

\section{Discussion}

\section{Principal Findings}

In this study, we showed that daily life gait speeds varied depending on the time of day and day of the week. Daily life gait speed showed greater variability than usual gait speed. The mean daily life gait speed was lower in participants with sarcopenia. When analyzing factors related to daily life gait speed, age and skeletal muscle mass of the lower limbs were significantly associated with mean daily life gait speed. The mean daily life gait speed was significantly and positively correlated with handgrip strength and appendicular skeletal muscle mass.

Walking speed is a valid and reliable measure for assessing the functional status of older adults [21], and identification of a slow walking speed is a simple approach to the diagnosis of frailty [22]. Gait speed at an individual's usual pace is known to be associated with survival in older adults [23] and has been widely used as a tool to predict adverse outcomes in community-dwelling older individuals, including falls, disability, institutionalization, and mortality $[9,24]$. However, one study demonstrated that usual gait speed does not represent daily life gait speed because it is not strongly related to daily gait speed [15]. Assessment of daily life gait speed showed that the men in our study walked significantly faster in the morning than during the rest of the day, and men with sarcopenia also walked faster in the morning. People may walk faster in the early morning because they usually have to go to work or attend social activities at a specified time. Participants in our study also walked faster on weekdays than on weekends. However, this difference was not significant in individuals with sarcopenia. This may be related to a decrease in the daily life gait speed of participants with sarcopenia, especially a decrease in the use of a fast gait speed. This is supported by the fact that men without sarcopenia often walked faster than their mean daily life gait speed, whereas participants with sarcopenia did not. As gait speed varied depending on the time of day and day of the week and the distribution of gait speed was very diverse, it would be difficult to accurately reflect the actual gait speed of individuals using one or two usual gait speed measurements obtained in the clinical setting. In fact, we found that the Pearson correlation coefficient between daily life gait speed and usual gait speed was negligible [25]. In addition, although the direct correlation between walking time and sarcopenia-related factors is not clear, people who spend more time walking tend to walk faster in their daily lives. An increase in walking-related exercise may be associated with a decrease in sarcopenia [26].

In this study, the mean value was used to represent the daily life gait speed to identify factors related to gait speed and to analyze the associations between gait speed and sarcopenia. Age and skeletal muscle mass of the lower limbs were significantly associated with the mean daily life gait speed. Reduced walking speed is associated with increasing age $[27,28]$. A decrease in the muscle mass of the lower limbs is also associated with a decrease in gait speed [29].

The participants in our study who had sarcopenia walked significantly more slowly in their daily lives than those without sarcopenia. This result was expected considering that patients with sarcopenia demonstrate various deteriorations in their physical performance [30,31]. Gait speed is a representative indicator of physical performance and is included in the diagnostic criteria for sarcopenia [6,7]. Diagnostic criteria commonly use the results of a 4- or 6-m usual gait speed test. Analyzing associations between daily life gait speed, muscle strength, and muscle mass, daily life gait speed also accurately reflects the sarcopenic state.

Advances in wearable devices have made it possible to continuously measure daily gait speed in the real world. As men usually wear belts in their daily lives, belt-type wearable devices can be used to measure daily life gait speed as naturally as possible over a long period. In fact, smart belt wearers can check their average walking speed on a daily, weekly, or monthly basis using a specific application. Therefore, older people would be able to identify a decrease in their physical performance in real life and, if so, would be able to visit health care providers for active intervention.

\section{Strengths and Limitations}

This study had several strengths. First, the analysis was performed using gait speed data measured more than 200,000 
times over a period of $\geq 10$ days. Therefore, the results reflect the actual gait speed of the participants more accurately than the current common practice of usual gait speed measured in a laboratory at only one point in time. Second, the skeletal muscle mass of participants was accurately measured by the standard method using DEXA, and it was confirmed that the skeletal muscle mass of the lower limbs had a statistically significant association with gait speed. Third, we found that walking speed could be measured continuously over a long period using a belt-type wearable device. On the basis of these results, it is feasible to carry out more precise longitudinal studies in the future, including studies focused on the prediction of a negative prognosis related to a change in walking speed.

This study has some limitations. First, we could not determine a causal relationship between skeletal muscle mass and gait speed because of the limitations inherent to the cross-sectional study design. A second limitation was that the study included only male participants. We chose male participants as Korean women who met our age criterion of $>50$ years do not usually wear belts, and we wanted to be certain that the participants could wear the device with ease. Accordingly, the findings observed in this study cannot be applied to women who usually do not use belts. To overcome this problem, we considered two solutions to this limitation. One was to improve the esthetic look of the belt so that women could wear it as a fashion accessory. The other was to place a plastic case containing the sensor, circuit, and battery in the same position as the buckle of the belt in the form of a clip.
Finally, we developed an algorithm for detecting and analyzing gait speed, but this has not been fully validated. However, a previous study reported that a method based on the detection of heel-strike events using a triaxial accelerometer to confirm that each step is suitable for measuring gait speed [32]. In our unpublished data, when comparing the gait data measured using WELT with that measured using video readings in 10 healthy adults, the mean step count was $95.9 \%$ (SD $4.2 \%$ ) consistent. The mean sensitivity of the WELT step detection algorithm was $87.9 \%$ (SD 2.8\%), and the mean positive predictive value was $92.4 \%$ (SD 3.3\%). However, there were no data regarding gait speed in patients with pathological gait patterns such as neurologic (Parkinson disease) or orthopedic conditions. Further studies are required to confirm the validity of wearable device-based gait speed measurements.

\section{Conclusions}

Diverse and accurate information can be obtained by measuring daily life gait speed using a wearable device. Daily life gait speed is significantly associated with age and skeletal muscle mass of the lower limbs. As gait speed is a representative indicator of physical performance, older individuals would be able to detect a decrease in physical performance in real life by checking their walking speed. In addition, as it is possible to measure daily gait speed continuously over a long period using a wearable device, daily life gait speed data will be available for use in future longitudinal studies. Further studies are needed, especially studies aimed at developing a method to accurately measure women's daily life gait speed as naturally as possible.

\section{Acknowledgments}

This study was supported by Seoul National University Bundang Hospital (SNUBH 14-2018-001). The funder of this study was not involved in study design, collection of data, interpretation of data, or writing of the manuscript.

\section{Conflicts of Interest}

SJK and HKR are cofounders of WELT Corp, Ltd. HYJ is a full-time employee of WELT Corp, Ltd. The other authors declare no conflicts of interest.

\section{References}

1. Kontis V, Bennett JE, Mathers CD, Li G, Foreman K, Ezzati M. Future life expectancy in 35 industrialised countries: projections with a Bayesian model ensemble. Lancet 2017 Apr;389(10076):1323-1335. [doi: 10.1016/s0140-6736(16)32381-9] [Medline: 28236464]

2. Mitnitski A, Collerton J, Martin-Ruiz C, Jagger C, von Zglinicki T, Rockwood K, et al. Age-related frailty and its association with biological markers of ageing. BMC Med 2015 Jul 13;13:161 [FREE Full text] [doi: 10.1186/s12916-015-0400-x] [Medline: 26166298]

3. Klein BE, Klein R, Knudtson MD, Lee KE. Frailty, morbidity and survival. Arch Gerontol Geriatr 2005;41(2):141-149. [doi: 10.1016/j.archger.2005.01.002] [Medline: 16085065 ]

4. Mach M, Watzal V, Hasan W, Andreas M, Winkler B, Weiss G, et al. Fitness-tracker assisted frailty-assessment before transcatheter aortic valve implantation: proof-of-concept study. JMIR Mhealth Uhealth 2020 Oct 15;8(10):e19227 [FREE Full text] [doi: 10.2196/19227] [Medline: 33055057]

5. Fried LP, Tangen CM, Walston J, Newman AB, Hirsch C, Gottdiener J, Cardiovascular Health Study Collaborative Research Group. Frailty in older adults: evidence for a phenotype. J Gerontol A Biol Sci Med Sci 2001 Mar;56(3):146-156. [doi: 10.1093/gerona/56.3.m146] [Medline: 11253156]

6. Chen L, Woo J, Assantachai P, Auyeung T, Chou M, Iijima K, et al. Asian Working Group for Sarcopenia: 2019 consensus update on sarcopenia diagnosis and treatment. J Am Med Dir Assoc 2020 Mar;21(3):300-307. [doi:

10.1016/j.jamda.2019.12.012] [Medline: 32033882]

7. Cruz-Jentoft AJ, Bahat G, Bauer J, Boirie Y, Bruyère O, Cederholm T, Writing Group for the European Working Group on Sarcopenia in Older People 2 (EWGSOP2), the Extended Group for EWGSOP2. Sarcopenia: revised European consensus 
on definition and diagnosis. Age Ageing 2019 Jan 01;48(1):16-31 [FREE Full text] [doi: 10.1093/ageing/afy169] [Medline: 30312372]

8. Hoogendijk EO, Afilalo J, Ensrud KE, Kowal P, Onder G, Fried LP. Frailty: implications for clinical practice and public health. Lancet 2019 Oct 12;394(10206):1365-1375. [doi: 10.1016/S0140-6736(19)31786-6] [Medline: 31609228]

9. van Kan GA, Rolland Y, Andrieu S, Bauer J, Beauchet O, Bonnefoy M, et al. Gait speed at usual pace as a predictor of adverse outcomes in community-dwelling older people an International Academy on Nutrition and Aging (IANA) Task Force. J Nutr Health Aging 2009 Dec;13(10):881-889. [doi: 10.1007/s12603-009-0246-z] [Medline: 19924348]

10. Cesari M, Kritchevsky SB, Penninx BW, Nicklas BJ, Simonsick EM, Newman AB. Prognostic value of usual gait speed in well-functioning older people--results from the Health, Aging and Body Composition Study. J Am Geriatr Soc Oct 2005 Oct;53(10):1675-1680. [doi: 10.1111/j.1532-5415.2005.53501.x] [Medline: 16181165]

11. Hardy SE, Perera S, Roumani YF, Chandler JM, Studenski SA. Improvement in usual gait speed predicts better survival in older adults. J Am Geriatr Soc 2007 Nov;55(11):1727-1734. [doi: 10.1111/j.1532-5415.2007.01413.x] [Medline: 17916121]

12. Li X, Dunn J, Salins D, Zhou G, Zhou W, Schüssler-Fiorenza RS, et al. Digital Health: Tracking physiomes and activity using wearable biosensors reveals useful health-related information. PLoS Biol 2017 Jan;15(1):e2001402 [FREE Full text] [doi: 10.1371/journal.pbio.2001402] [Medline: 28081144]

13. Kim K, Gollamudi SS, Steinhubl S. Digital technology to enable aging in place. Exp Gerontol 2017 Feb;88:25-31. [doi: 10.1016/j.exger.2016.11.013] [Medline: 28025126]

14. Mueller A, Hoefling HA, Muaremi A, Praestgaard J, Walsh LC, Bunte O, et al. Continuous digital monitoring of walking speed in frail elderly patients: noninterventional validation study and longitudinal clinical trial. JMIR Mhealth Uhealth 2019 Nov 27;7(11):e15191 [FREE Full text] [doi: 10.2196/15191] [Medline: $\underline{\text { 31774406] }}$

15. Van Ancum JM, van Schooten KS, Jonkman NH, Huijben B, van Lummel RC, Meskers CG, et al. Gait speed assessed by a 4-m walk test is not representative of daily-life gait speed in community-dwelling adults. Maturitas 2019 Mar;121:28-34. [doi: 10.1016/j.maturitas.2018.12.008] [Medline: 30704562]

16. Grieve DW, Gear RJ. The relationships between length of stride, step frequency, time of swing and speed of walking for children and adults. Ergonomics 1966 Sep;9(5):379-399. [doi: 10.1080/00140136608964399] [Medline: 5976536]

17. Guest R, Miguel-Hurtado O, Stevenage S, Black S. Exploring the relationship between stride, stature and hand size for forensic assessment. J Forensic Leg Med 2017 Nov;52:46-55. [doi: 10.1016/j.jflm.2017.08.006] [Medline: 28865387]

18. Choi J, Kim K, Choi Y, Ahn S, Kang E, Oh H, et al. Comparison of multidimensional frailty score, grip strength, and gait speed in older surgical patients. J Cachexia Sarcopenia Muscle 2020 Apr;11(2):432-440 [FREE Full text] [doi: 10.1002/jcsm.12509] [Medline: 31912668]

19. Guerri S, Mercatelli D, Gómez MP, Napoli A, Battista G, Guglielmi G, et al. Quantitative imaging techniques for the assessment of osteoporosis and sarcopenia. Quant Imaging Med Surg 2018 Feb;8(1):60-85 [FREE Full text] [doi: 10.21037/qims.2018.01.05] [Medline: 29541624]

20. Lee SY, Ahn S, Kim YJ, Ji MJ, Kim KM, Choi SH, et al. Comparison between dual-energy x-ray absorptiometry and bioelectrical impedance analyses for accuracy in measuring whole body muscle mass and appendicular skeletal muscle mass. Nutrients 2018 Jun 07;10(6):1-14 [FREE Full text] [doi: 10.3390/nu10060738] [Medline: 29880741]

21. Middleton A, Fritz SL, Lusardi M. Walking speed: the functional vital sign. J Aging Phys Act 2015 Apr;23(2):314-322 [FREE Full text] [doi: 10.1123/japa.2013-0236] [Medline: 24812254]

22. Castell M, Sánchez M, Julián R, Queipo R, Martín S, Otero A. Frailty prevalence and slow walking speed in persons age 65 and older: implications for primary care. BMC Fam Pract 2013;14:86 [FREE Full text] [doi: 10.1186/1471-2296-14-86] [Medline: 23782891]

23. Studenski S, Perera S, Patel K, Rosano C, Faulkner K, Inzitari M, et al. Gait speed and survival in older adults. J Am Med Assoc 2011 Jan 5;305(1):50-58 [FREE Full text] [doi: 10.1001/jama.2010.1923] [Medline: 21205966]

24. Verghese J, Holtzer R, Lipton RB, Wang C. Quantitative gait markers and incident fall risk in older adults. J Gerontol A Biol Sci Med Sci 2009 Aug;64(8):896-901 [FREE Full text] [doi: 10.1093/gerona/glp033] [Medline: 19349593]

25. Mukaka MM. Statistics corner: A guide to appropriate use of correlation coefficient in medical research. Malawi Med J 2012 Sep;24(3):69-71 [FREE Full text] [Medline: 23638278]

26. Yoo S, No M, Heo J, Park D, Kang J, Kim SH, et al. Role of exercise in age-related sarcopenia. J Exerc Rehabil 2018 Aug;14(4):551-558 [FREE Full text] [doi: 10.12965/jer.1836268.134] [Medline: 30276173]

27. Himann JE, Cunningham DA, Rechnitzer PA, Paterson DH. Age-related changes in speed of walking. Med Sci Sports Exerc 1988 Apr;20(2):161-166. [doi: 10.1249/00005768-198820020-00010] [Medline: 3367751]

28. Busch TD, Duarte YA, Nunes D, Lebrão ML, Naslavsky MS, dos Santos Rodrigues A, et al. Factors associated with lower gait speed among the elderly living in a developing country: a cross-sectional population-based study. BMC Geriatr 2015 Apr 01;15:35 [FREE Full text] [doi: 10.1186/s12877-015-0031-2] [Medline: 25880124]

29. Beavers KM, Beavers DP, Houston DK, Harris TB, Hue TF, Koster A, et al. Associations between body composition and gait-speed decline: results from the Health, Aging, and Body Composition study. Am J Clin Nutr 2013 Mar;97(3):552-560 [FREE Full text] [doi: 10.3945/ajcn.112.047860] [Medline: 23364001] 
30. Cesari M, Landi F, Vellas B, Bernabei R, Marzetti E. Sarcopenia and physical frailty: two sides of the same coin. Front Aging Neurosci 2014;6:192 [FREE Full text] [doi: 10.3389/fnagi.2014.00192] [Medline: 25120482]

31. Dutta C, Hadley EC, Lexell J. Sarcopenia and physical performance in old age: overview. Muscle Nerve Suppl 1997;5:5-9. [Medline: 9331374]

32. Fortune E, Lugade V, Morrow M, Kaufman K. Validity of using tri-axial accelerometers to measure human movement Part II: Step counts at a wide range of gait velocities. Med Eng Phys 2014 Jun;36(6):659-669 [FREE Full text] [doi: 10.1016/j.medengphy.2014.02.006] [Medline: 24656871]

\section{Abbreviations \\ DEXA: dual-energy x-ray absorptiometry \\ WELT: wearable smart belt}

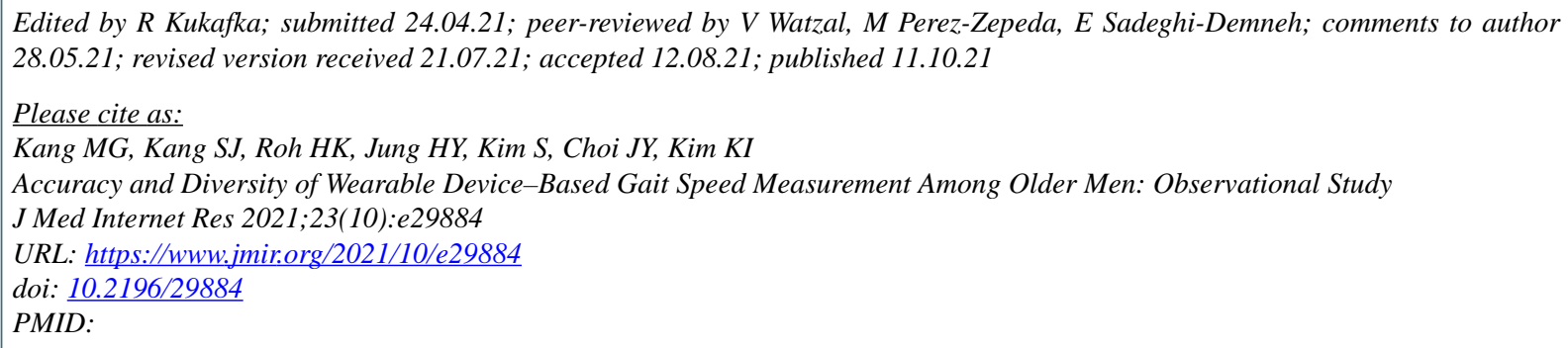

CMin-gu Kang, Seong-Ji Kang, Hye-Kang Roh, Hwa-Young Jung, Sun-wook Kim, Jung-Yeon Choi, Kwang-il Kim. Originally published in the Journal of Medical Internet Research (https://www.jmir.org), 11.10.2021. This is an open-access article distributed under the terms of the Creative Commons Attribution License (https://creativecommons.org/licenses/by/4.0/), which permits unrestricted use, distribution, and reproduction in any medium, provided the original work, first published in the Journal of Medical Internet Research, is properly cited. The complete bibliographic information, a link to the original publication on https://www.jmir.org/, as well as this copyright and license information must be included. 